\title{
Influence of cardiopulmonary bypass on some hematological and antioxidants parameters in cardiac patients and associated diseases
}

\author{
Mostafa Aboudiah $^{1}$, Hesham Abdelrachied ${ }^{1}$, Diaa Ahmed ${ }^{1}$, and Omar Dawoud ${ }^{2}$ \\ ${ }^{1}$ Al-Azhar University Faculty of Science \\ ${ }^{2}$ Cairo University Kasr Alainy Faculty of Medicine
}

October 29, 2020

\begin{abstract}
Abstract Purpose: Exploring those possibility effect of (CPB); cardiopulmonary bypass on definite cardiovascular works and antioxidants in patients with open-heart and connected sicknesses. Methods: Twenty fit individuals also eighty patients starting with heart-surgery and cohorted ailments Similarly as diabetes, hepatitis furthermore renal failure were presented of the impact of CPB. Certain cardiac function Besides antioxidants were measured. Results: a huge diminishing in RBCs count, Hb. While, a critical build was recorded alongside WBCs count, MDA, NO level Also (GPX) activity in cardiovascular patients connected with above-mentioned illnesses contrasting to their control groups on the 1st and 2nd post-operative days.
\end{abstract}

Influence of cardiopulmonary bypass on some hematological and antioxidants parameters in cardiac patients and associated diseases.

Authors: Aboudiah, M. E.1, ABDELRACHIED, H. G. 1., Farrag D. A. 1, Omar Dawoud. 2.

1. Zoology and Entomology department, Faculty of Science, Al-Azhar University, Nasr City, Cairo, Egypt.

2. Cardio-thoracic surgery unit, Kasr El-Aini hospital, Faculty of Medicine, Cairo University.

Corresponding author*: mostafaelsayed5@gmail.Com

Abstract

Purpose: Exploring those possibility effect of (CPB); cardiopulmonary bypass on definite cardiovascular works and antioxidants in patients with open-heart and connected sicknesses.

Methods: Twenty fit individuals also eighty patients starting with heart-surgery and cohorted ailments Similarly as diabetes, hepatitis furthermore renal failure were presented of the impact of CPB. Certain cardiac function Besides antioxidants were measured.

Results: a huge diminishing in RBCs count, Hb. While, a critical build was recorded alongside WBCs count, MDA, NO level Also (GPX) activity in cardiovascular patients connected with above-mentioned illnesses contrasting to their control groups on the $1^{\text {st }}$ and $2^{\text {nd }}$ post-operative days.

K E Y W O R D S

Cardiopulmonary bypass, Diabetes, Hepatitis, renal failure, hematology, Antioxidants.

1. Introduction

Precisely actuated myocardial ischemia, notwithstanding transient, incites the release of possibly hurtful oxygen free radicals Likewise and just a many-sided combustible course that advances to those destinations 
of the tissue harm. ${ }^{1}$ generally cardiopulmonary bypass (CPB) is a plan that prohibitively choose those limit about heart and lungs throughout activity additionally eventually the examining saving those flowing library for blood and the oxygen supply of the constitution. Cardiopulmonary sidestep (CPB) technobabble need been another advancement secured close by therapeutic science that allowed cardiovascular specialists with choose a couple of was irksome open-heart medical procedures for instance, coronary diversion medical procedure, valve substitution, fix shed about A rate considerable aneurysm and fix shed from guaranteeing natural anomalies. ${ }^{2}$ Cardiopulmonary sidestep (CPB) will be associated with the incitation for various coagulation pathways, supportive of irritation, key falls, Also adjusted redox state. Hemolysis, ischemic reperfusion injury, additionally neutrophil incitation that make throughout $\mathrm{CPB}$ need a basic part in the course of action from asserting oxidative pressure. This condition could change the clinical results toward impacting those limits from asserting organs for instance, with the end goal that lungs besides kidneys Also chiefly myocardium. ${ }^{3}$ The greater part basic medical procedure and explicitly cardiovascular medical procedure are a trial of the hematopoietic structure. The use about cardiopulmonary bypass (CPB), drain (all through and after medical procedure), unremitting blood investigations (previously, throughout also then a while later medical procedure), fix weakening, basic development about intravascular volume, mechanical injury of platelets, remedial hypothermia, co-morbidities, the use of anticoagulant additionally platelets drugs (previously, throughout What's all the more after medical procedure), bonding of blood items, establishment basic movements in the three significant cell division parts of the hematopoietic framework. ${ }^{4,2}$ accounted that cardiopulmonary sidestep (CPB) convinced differences on hematological What's more hemorrhagic factors Likewise a delayed consequence for hematological and hemorrhagic boundaries for instance, hemoglobin fixation, complete blood check, PT, moreover international normalized ratio (INR) were tried using standard lab frameworks going before activity (preoperative) additionally then subsequently first, third, other than seventh long stretches of activity. Glutathione peroxidase and superoxide dismutase express an ordinary likewise sturdy extend over development throughout medical procedure (40 moreover 30\%, correspondingly), getting back with benchmark characteristics $24 \mathrm{~h}$ after medical procedure. Also ${ }^{5}$ found that degree oxidative pressure impacts a sum for endogenous antioxidants and markers of cellular actuation. ${ }^{6}$ accounted that open-heart medical procedures did with heart-lung machine activate oxidative pressure. This situation will be almost associated with wealth ROS arrangement. Oxidative reaction reasons hurt in the cell limit likewise may assemble the challenges throughout or then subsequently coronary conduit evade joining (CABG) medical procedure. Cell fortification particles may explicitly react for delicate revolutionaries likewise may degenerate.

\section{MATERIALS AND METHODS.}

\section{1. Test plan.}

Hundred people (twenty healthy people and eighty of coronary illness and sicknesses co-collaborated required conceded with cardiothoracic medical procedure unit in Kasr El-Aini emergency clinic (Faculty of Medicine, Cairo University) suffering beginning with cardiovascular diseases just/or associated with some different contaminations as: diabetes mellitus sort I or kind II, hepatitis c and/or hepatitis b besides renal failure who were exposed to go through heart medical procedures; open-heart medical procedures: for instance, coronary supply route sidestep joining; (CABG), (valve fix or substitution), and so on...

\section{2. Hematological parameters.}

Blood examples were collected in a vial containing anticoagulant agent, for example, ethylene diamine tetra acidic corrosive (EDTA). Erythrocytes (RBCs) check, hemoglobin (Hb) focus, leucocytes (WBCs) tally, may have been assessed by cell counter (CBC instrument; Diagon_ Ltd, D-cell 60 auto hematology Analyzer).

\subsection{Antioxidants biomarker.}

Lipid peroxidation change will be settled by the thiobarbituric corrosive (TBA) framework which assesses the malondialdehyde creation (MDA) concurring to7. Those test for GSH activity may have been settled as expressed by the framework for8. Nitric oxide level may have been controlled as expressed by the system for9. Which estimated using business unit purchased from Biomed indicative organization, may have been 
assessed by science analyzer (Photometer 5010 V5+, Robert RIELE GmbH and Co KG, Germany).

2. 4. Statistical analysis.

Graph Pad Prism 8.0.2 was utilized for information the executives. Two-way ANOVA looked at the methods for free gatherings. L.S.D.; (Least huge contrast) test was done to decide whether there may have been a basic qualification among the previously mentioned sets and their controls where $\mathrm{P}$-value $<0.05$ was critical increment. The information was expressed as mean \pm S.D. Differences were recognized measurably incredibly enormous at $(\mathrm{P}<0.001)$ and factually important during $(\mathrm{P}<0.05)$.

\section{Results.}

Calculable information in tables (1-6) showed a vital decrease (P [?] 0.05) to hemoglobin concentration among groups, ages besides interactions on the first and second days postoperatively. Additionally, by using L.S.D. Hemoglobin fixation uncovered huge abatement (P [?] 0.05) in all gatherings (heart, diabetic, hepatic and renal disappointment patients) individually contrasting with control gatherings.

Tables (7, 8 and 9) demonstrated gigantic decay (P [?] 0.05) in red blood corpuscles (RBCs) number between bunches on the first day after activity. Alternately, non-huge distinction was recorded in RBCs tally among ages and connections. where by utilizing L.S.D. Red blood corpuscles number uncovered critical decline (P [?] 0.05) in all gatherings; heart, diabetic, hepatic and renal disappointment patients separately contrasting with control gatherings.

From measurable investigation in tables $(10,11$ and 12$)$ there were decreased altogether $(\mathrm{P}$ [?] 0.05$)$ over red blood corpuscles number between bunches likewise collaborations on second day after activity. Conversely, non-huge distinction was recorded in RBCs tally between ages. Where by using L.S.D. Red blood corpuscles number uncovered huge reduction (P [?] 0.05) in all gatherings; heart, diabetic and renal disappointment patients separately contrasting with control gatherings, aside from cardiovascular patients related with hepatic sickness indicated non-critical distinction when stood out from control gathering.

ANOVA of tables $(13,14$ and 15) uncovered non-huge contrast in all out number of leucocytes among ages and communications on the main day after activity. While a critical expanding ( $\mathrm{P}$ [?] 0.05) was seen between gatherings. where by using L.S.D. Complete leucocytes check indicated critical expanding (P [?] 0.05) in all gatherings; heart, diabetic, hepatic and renal disappointment patients individually contrasting with control gatherings.

Information in tables (16, 17 and 18) demonstrated non-huge distinction for absolute leucocytes tally between ages. On contrast a huge increment (P [?] 0.05) was seen among gatherings and associations on the second day after activity. where by using L.S.D. All out leucocytes check recorded important growing (P [?] 0.05) in all gatherings once differentiated to their controller sets.

Tables (19, 20 and 21) demonstrated non-critical contrast in Malondialdehyde (MDA) level between ages, while huge rising (P [?] 0.05) for Malondialdehyde (MDA) level among gatherings and collaborations on the first day after activity. by means of using L.S.D. Malondialdehyde (MDA) level uncovered a critical increment in all gatherings contrasting with control gatherings. Non-critical contrast in Malondialdehyde (MDA) level was among ages and collaborations. In the contrary side, an expanding altogether (P [?] 0.05) in Malondialdehyde (MDA) level was recorded between bunches on the second day after activity, where by using L.S.D. Malondialdehyde (MDA) level introduced critical increment (P [?] 0.05) in cardiovascular patients and heart patients related with (hepatic, diabetic and renal disappointment) sicknesses separately contrasted with control bunches in tables (22, 23 and 24).

Tables (25 - 30) displayed vital grow (P [?] 0.05) in nitric oxide level among gatherings and cooperation on the first additionally second day postoperatively. by using L.S.D. Nitric oxide (NO) level watched critical increment (P [?] 0.05) in heart patients and cardiovascular patients related with (hepatic or diabetic or renal disappointment) infection contrasting with control gatherings. 
Statistical outcomes in tables (31-36) uncovered critical contrast (P [?] 0.05) in glutathione peroxidase actiuation between gatherings while non-huge change was recorded among ages and connections on the $1^{\text {st }}$ and second day after surgery. By using L.S.D. Glutathione peroxidase (GPX) demonstrated non-huge change in cardiovascular patients. On contrast a vital extend (P [?] 0.05) over glutathione peroxidase incitation may have been recognized on cardiovascular patients related with (hepatic, diabetic and renal disappointment) infections contrasting with the benchmark groups.

\section{Discussion.}

The outcomes obviously indicated significant contrasts in a few hematological and draining factors Likewise altered hemoglobin level, blood cells number, INR moreover PT in patients go through cardiovascular medical procedure with CPB in going before postoperative days conversely with patients going through cardiovascular medical procedure without $\mathrm{CPB} .{ }^{2,10}$ detailed that leukocyte actiuation happen because of a cardiopulmonary evade $(\mathrm{CPB})$ usage during heart medical procedure, besides around different causes, actiuate organ debilitation in view of extended leukocyte staffing in different organs. ${ }^{11}$ announced that various degrees about bothersome combustible responses, stretching out from a diminished measure of extreme fundamental combustible response to extra serious multi-organ harm due to on-pump cardiothoracic medical procedure. Outrageous oxidative pressure due to production of oxidation byproducts created throughout ischemia likewise reperfusion may make Concerning outline an eventual outcome about cardiovascular surgery. ${ }^{12}$ informed those fundamental effects regarding cardiopulmonary evade (CPB) that lead to harmed directly in the myocardium Additionally lead to critical proportion of the restricting results ${ }^{13}$ recommended that on-pump strategy accommodates tremendous danger will a foundational irritation What's more oxidative effort over the off-pump procedure. Clarification of these observations may make related to not many pernicious events occurring throughout $\mathrm{CPB}$ which are whichever material-dependent (created by presentation from guaranteeing blood will non-physiologic surfaces moreover states all through the extracorporeal dissemination, ECC) or material-independent (brought about by scrutinizing careful trauma). ${ }^{14,} 15$ in like manner watched a diminished done RBCs number all through the essential 3 to 4 days after medical procedure. Then again, over CPB-helped cardiovascular medical procedure hem dilution for CPB will be recognized valuable to consequence of medical procedure; hem dilution can't make avoided Concerning representation from Numerous concentrations for viewpoint. The past impacts of huge segments research clearly demonstrated that hem dilution provoked sickliness might be typical over CPB-helped cardiovascular medical procedure. Thus, it need been suggested that mechanical qualities all through extracorporeal circulation may reason finish obliteration of the RBC, speedy on the other hand Postponed. At last, CPB is additionally alluded to on make basic movements in the mechanical properties about $\mathrm{RBCs}^{8}$, for instance, with the end goal that lessening their deformability What's more surface charge besides growing their delicacy likewise agree.

\section{REFERENCES}

1. Castillo CA, Angela MM, Olalla ME, et al . Comparative Analysis of Antioxidant Defense During On-Pump and Off-Pump Cardiac Surgery. Rev Esp Cardiol. 2005; 58(7):822-9.

2. Aftabuddin MD, Rajbhandhari N, Rahman Z, et al. Cardiopulmonary Bypass Induced Hematological Changes in Patients Undergoing Cardiac Surgery. Bangladesh Heart Journal 2015; 30(2): 53-57).

3. Mustafa Z, Gustavo G, Suleiman S, et al. Cardiopulmonary bypass and Oxidative Stress. Oxidative Medicine and Cellular Longevity, 2015; 8:1-10.

4. Wang A, Bashore TM. Hematologic Disorders after Cardiac Surgery. Valvular Heart Disease Book (2009); 432-35.

5. Sadowska I E, Chris RL, Frans JV, et al. Antioxidant defense during cardiopulmonary bypass surgery. Euro J Cardio-thoracic Surg. (2005); 27:611-616.

6. Turker F, Dogan A, Ozan G, et al. Change in Free Radical and Antioxidant Enzyme Levels in the Patients Undergoing Open Heart Surgery with 1783728.Cardiopulmonary Bypass. Oxidative Medicine and Cellular Longevity, (2016);1-5.

7. Draper HH, Hadley M. malondialdehyde determination as index of lipid per oxidation. Methods Enzymology. 1990; 186:421-31. 
8. Beutler E, Duron O, Kelly BM. Improved method for the determination of blood glutathione. Journal Laboratory Clinical Medicine. 1963; 61(5):882-888.

9. Montgomery HAC, Dymock JF. The determination of nitrite in water. Analyst. 1961; 86:414-416.

10. Rossaint J, Berger C, Van Aken H, et al. Cardiopulmonary Bypass during Cardiac Surgery Modulates Systemic Inflammation by Affecting Different Steps of the Leukocyte Recruitment Cascade. PLoS ONE (2012);7(9): e45738.

11. Jouybar R, Kabgani H, kamalipour H, et al The Effect of Ascorbic Acid on Inflammatory Response in Perioperative Coronary Artery Bypass Grafting Surgery. Int. Cardiovasc. Res. J. (2012); 6(1):13-17.

12. Luyten RC, van Overveld JF, De Backer AL, et al . Antioxidant defense during cardiopulmonary bypass surgery. European Journal Cardio-thoracic Surgery. (2005); 27: 611-616.

13. Wan S, LeClerc JL, Vincent JL. Inflammatory response to cardiopulmonary bypass:

mechanism involved and possible therapeutic strategies. Chest.1997; 112: 676-92.

Elgebaly SA, Houser SL, el Kerm AF, et al. Evidence of cardiac inflammation after open

heart operations. Ann Thorac Surg.1994; 57:391-6.

Salama A, Hugo F, Heinrich D, et al. Deposition of terminal C5b-9 complement

complexes during cardiopulmonary bypass. N Engl J Med 1988; 318:408-20. 